\title{
Clinical and molecular genetic risk determinants in adult long QT syndrome type 1 and 2 patients
}

\section{Koponen et al. Follow-up of adult LQTS patients}

\author{
Mikael Koponen ${ }^{1 *}$ (D, Aki S. Havulinna ${ }^{2}$, Annukka Marjamaa', Annukka M. Tuiskula ${ }^{3}$, Veikko Salomaa², \\ Päivi J. Laitinen-Forsblom ${ }^{4}$, Kirsi Piippo ${ }^{5}$, Lauri Toivonen' ${ }^{1}, K^{2}$ mo Kontula ${ }^{3}$, Matti Viitasalo ${ }^{1}$ and Heikki Swan ${ }^{1}$
}

\begin{abstract}
Background: Long QT syndrome (LQTS) is an inherited cardiac disorder predisposing to sudden cardiac death (SCD). We studied factors affecting the clinical course of genetically confirmed patients, in particular those not receiving $\beta$-blocker treatment. In addition, an attempt was made to associate risk of events to specific types of KCNQ1 and KCNH2 mutations.
\end{abstract}

Methods: A follow-up study covering a mean of $18.6 \pm 6.1$ years was conducted in 867 genetically confirmed LQT1 and LQT2 patients and 654 non-carrier relatives aged 18-40 years. Cox regression models were used to evaluate the contribution of clinical and genetic risk factors to cardiac events.

Results: In mutation carriers, risk factors for cardiac events before initiation of $\beta$-blocker included LQT2 genotype (hazard ratio $[H R]=2.1, p=0.002)$, female gender $(H R=3.2, p<0.001)$, a cardiac event before the age of 18 years $(H R=5.9, p<0.001)$, and QTc $\geq 500 \mathrm{~ms}(\mathrm{vs}<470 \mathrm{~ms}, \mathrm{HR}=2.7, p=0.001)$. LQT1 patients carrying the KCNQ1 D317N mutation were at higher risk ( $H R=3.0-3.9, p<0.001-0.03)$ compared to G589D, c.1129-2A > G and other KCNQ1 mutation carriers after adjusting for gender, QTC duration, and cardiac events before age 18. KCNH2 C.453delC, L552S and R176W mutations associated with lower risk (HR $=0.11-0.23, \mathrm{p}<0.001)$ than other KCNH2 mutations.

Conclusions: LQT2 (compared to LQT1), female gender, a cardiac event before age 18, and long QT interval increased the risk of cardiac events in LQTS patients aged 18 to 40 years. The nature of the underlying mutation may be associated with risk variation in both LQT1 and LQT2. The identification of high-risk and low-risk mutations may enhance risk stratification.

Keywords: Long QT syndrome, Cardiac arrhythmia, Risk stratification, $\beta$-blocker, Implantable cardioverterdefibrillator

\section{Background}

Long QT syndrome (LQTS) is a hereditary cardiac disorder characterized by impaired repolarization properties of cardiomyocytes which predispose to ventricular arrhythmias, syncope and sudden cardiac death (SCD). [1] A total of 16 genes associate with LQTS, and mutations in KCNQ1 or KCNH2 genes cause the most

* Correspondence: mikael.koponen@helsinki.fi

${ }^{1}$ Heart and Lung Center, Helsinki University Central Hospital, Helsinki, Finland Full list of author information is available at the end of the article common subtypes LQT1 and LQT2, respectively. [2] According to recent ESC guidelines, $\beta$-blocker treatment should be initiated if QTc is prolonged, and it may be useful even with normal QTc. [3, 4] Implantable cardioverter-defibrillator (ICD) should be applied in more severe cases. $[5,6]$

Presently, genotype and mutation location can be applied as a part of risk stratification. [7-11] As the number of molecularly tested mutation carriers increases, mutation-specific assessment might enable more individually tailored patient management strategies. [12] 
However, the data available for mutation-specific risk stratification are still limited. [12-16] Previous studies assessing the clinical course in genotyped subjects have included children $[7,8]$ or patients treated with $\beta$ blockers. [9-11, 17-19] In LQTS, the risk associated with gender and genotype is age-related, [10, 11, 17, 19] and $\beta$-blocker treatment impacts disparate subgroups of patients differently. [17-19] In the current study, the clinical course without $\beta$-blocker treatment was explored in genotyped LQT1 and LQT2 patients aged 18-40 years. In addition, we studied the association of six different LQTS-causing mutations with prognosis of the LQT1 and LQT2 patients.

\section{Methods}

\section{Study population}

The study population was drawn from the Finnish Inherited Arrhythmic Disorder Research Registry established in 1991 and comprising over 4000 molecularly tested subjects. The inclusion criteria were 1) genetically confirmed KCNQ1 or KCNH2 mutation, or genetically confirmed non-carrier status of the family-specific LQTS mutation, 2) and the age of more than 18 years at follow-up end. A questionnaire (Additional file 1) was sent to the study subjects and collected data included occurrence of syncope, setting in which syncope occurred, and data regarding $\beta$-blocker therapy. Compliance was defined as forgetting or not taking medication once a month or more often. The decision whether to initiate $\beta$-blocker therapy was made by the treating physician upon establishment of the diagnosis.

Data of all deaths during the follow-up were obtained from Statistics Finland by means of social security number search. ICD and pacemaker implantations, and left cardiac sympathetic denervations (LCSD) were identified using the Finnish Hospital Discharge Register (National Institute for Health and Welfare). Medical records were acquired for patients who had device therapy, underwent LCSD, suffered an aborted cardiac arrest (ACA), or died. Collected ICD data included implantation indications, complications, revisions, and ICD discharges. Autopsy documents of patients who died during the follow-up were evaluated. The study was approved by the Ethical Review Committee of Helsinki University Hospital, and a written informed consent was obtained from the study subjects. The Ministry of Social Affairs and Health consented for the participation of deceased subjects.

The follow-up study started from the age of 18 years and ended when the subject 1) returned the questionnaire, 2) turned 40 years [to avoid the effect of acquired cardiac disease] or 3) was deceased, which ever occurred first. The end point for statistical analyses was cardiac event comprising LQTS-related syncope, ACA, appropriate ICD shock, or SCD. LQTS-related syncope was defined as a transient loss of consciousness that was abrupt in onset and offset, and triggered by one of the following factors: swimming, other sports, loud noise, or startle, to avoid inclusion of vasovagal events. [9] Resuscitation events that required external defibrillation were defined as ACA. A death was regarded as being SCD if it was abrupt in onset without evident cause if witnessed, or was not explained by any other cause if it occurred in an unwitnessed setting such as sleep.

Direct DNA sequencing and restriction enzyme assays were used in identification of $K C N Q 1$ and $K C N H 2$ mutations as previously described. [20, 21] Mutations were categorized by mutation type as missense or non-missense (nonsense, frameshift, splice site, insertion or deletion) mutations, and by their location as described previously. $[10,11]$ Patients carrying more than one LQTS mutation $(n=7)$ were excluded from the comparison of the clinical characteristics and the multivariate risk analyses, but were included in the sections depicting device therapy. The specific single mutations included in the final study population are detailed in Additional file 2: Table S1. LQT1 Finnish founder (FF) mutations KCNQ1 G589D and KCNQ1 c.1129-2A > G, and LQT2 FF mutations $K C N H 2$ R176W and $K C N H 2$ L552S were combined to form the FF mutation population for LQT1 and LQT2, respectively. Non-carrier family members of the familial KCNQ1 and $\mathrm{KCNH} 2$ mutations served as the comparison group. All study subjects were of Finnish origin.

\section{Statistical analyses}

Clinical characteristics were analyzed using chi-square and Fisher's exact tests for categorical, and Wilcoxon rank-sum and Kruskal-Wallis one-way ANOVA tests for continuous variables. Kaplan-Meier methods were used to depict the cumulative incidence rate (=cumulative probability) of first cardiac event after the age of 18 years by genotype, gender, QTc interval, and mutation. The QTc cut-offs used were based on previous LQTS studies. $[4,7,18]$ The significance of the differences was tested by the log-rank test. Multivariate Cox proportional hazards regression models were used to evaluate the independent contribution of genetic and clinical risk factors to first cardiac event after 18 years of age. Survival was also evaluated by assessing incidence rates of first cardiac events per person-years. All cumulative incidence graphs, log-rank tests, and primary Cox regression and incidence rate analyses were censored at the initiation of $\beta$-blocker medication. Secondary Cox regression and incidence rate analyses (Medical Treatment paragraph) were carried out including the follow-up time with timedependent $\beta$-blocker medication. No violation of the proportional hazards assumption was detected as tested by log-log graphs. A separate QTc missing covariate was used for mutation carriers whose QTc data were 
unavailable $(n=35)$. No statistically significant interactions were discovered in interaction term analyses. All Cox regression models were adjusted for gender, QTc duration, cardiac events before the age of 18 , and family membership using robust sandwich estimators. Statistical analyses were carried out using SPSS version 22 . A 2 -sided $p$-value $\leq 0.05$ was interpreted as statistically significant.

\section{Results}

A total of 2723 subjects fulfilled the inclusion criteria. The final study population $(n=1521)$ consisted of 14 subjects who died during the follow-up, 1495 subjects who responded (55\%) to the inquiry, and additional 12 subjects with device therapy drawn from the Hospital Discharge Register. The study population comprised 867 LQTS mutation carriers (617 KCNQ1, $243 \mathrm{KCNH2}$, and seven with $>1 K C N Q 1$ or $K C N H 2$ mutation), and 654 non-carrier relatives.

The final study cohort had 263 families, and 190 (22\%) of the mutation carriers were probands. The total follow-up time without $\beta$-blocker medication in a subgroup of 1420 subjects was $18.6 \pm 6.0$ years. There were 285 subjects who had $\beta$-blocker medication at some point of the study, and the mean follow-up time with medication was $6.2 \pm 5.4$ years. Nonresponders $(n=$ $2723-1521=1202$ ) had a higher proportion of males than subjects of the final study population $(52 \%$ vs $36 \%$, $p<0.001)$. There was no difference in the proportion of LQTS subtypes, or mean QTc duration between these two groups.

\section{Clinical characteristics}

The characteristics of the patients with a single mutation are shown in Table 1 and with more than one mutation in Additional file 2: Table S2. Characteristics of the non-carrier relatives are presented in Additional file 2: Table S3. Altogether seven of the ten deaths in mutation carriers, and none of the four deaths in non-carrier relatives were arrhythmia-related. Among mutation carriers seven (1\%) suffered a SCD and eight (1\%) at least one ACA. In LQT1 2\% and in LQT2 3\% of the cardiac

Table 1 Characteristics of the mutation carriers at the age of 18-40 years ${ }^{a}$

\begin{tabular}{|c|c|c|c|c|c|c|c|c|c|c|}
\hline & \multirow{2}{*}{\multicolumn{2}{|c|}{ All patients }} & \multicolumn{4}{|l|}{ LQT1 } & \multicolumn{4}{|l|}{ LQT2 } \\
\hline & & & \multicolumn{2}{|l|}{ Non-FF } & \multicolumn{2}{|l|}{$\mathrm{FF}$} & \multicolumn{2}{|l|}{ Non-FF } & \multicolumn{2}{|l|}{$\mathrm{FF}$} \\
\hline & LQT1 & LQT2 & D317N & Other & G589D & c. $1129-2 A>G$ & c.453delC & Other & L552S & R176W \\
\hline N (\%) & $617(72)$ & $243(28)$ & $20(3)$ & $72(12)$ & $453(73)$ & $72(12)$ & $23(10)$ & $61(25)$ & $73(30)$ & $86(35)$ \\
\hline Female & $396(64)$ & $157(65)$ & $17(85)$ & $48(67)$ & $282(62)$ & $49(68)$ & $11(48)_{a, b}{ }^{c}$ & $33(54)_{a}$ & $56(77)_{\mathrm{b}}$ & $57(66)_{a, b}$ \\
\hline Age, y & $36.3 \pm 6.2$ & $36.1 \pm 6.8$ & $36.5 \pm 5.5$ & $35.5 \pm 7.2$ & $36.3 \pm 6.1$ & $37.4 \pm 5.7$ & $35.1 \pm 8.2$ & $35.2 \pm 7.1$ & $37.0 \pm 6.0$ & $36.3 \pm 6.9$ \\
\hline $\mathrm{QTC}, \mathrm{ms}$ & $467 \pm 40$ & $465 \pm 41$ & $492 \pm 51_{a}{ }^{c}$ & $473 \pm 43_{b}$ & $465 \pm 40_{b}$ & $466 \pm 28_{b}$ & $466 \pm 39 a, b, c$ & $487 \pm 45_{a}$ & $466 \pm 42_{b}$ & $448 \pm 29_{c}$ \\
\hline Proband & $112(18)_{1}^{b}$ & $78(32)_{2}$ & $1(5)$ & $18(25)$ & $76(17)$ & $17(24)$ & $0_{a}$ & $34(56)_{b}$ & $22(30)_{c}$ & $22(26)_{c}$ \\
\hline$\beta$-blocker & $184(30)$ & $65(27)$ & $11(55)_{a}$ & $30(42)_{a, b}$ & $126(28)_{a, b}$ & $17(24)_{b}$ & $7(30)_{a, b}$ & $31(51)_{a}$ & $14(19)_{\mathrm{b}}$ & $13(15)_{b}$ \\
\hline $\mathrm{ICD}$ & $9(2)_{1}$ & $11(5)_{2}$ & $1(5)$ & $3(4)$ & $5(1)$ & 0 & $0_{a, b}$ & $9(15)_{a}$ & $2(3)_{a, b}$ & $\mathrm{O}_{\mathrm{b}}$ \\
\hline Pacemaker & $5(1)_{1}$ & $8(3)_{2}$ & 0 & 0 & $3(1)$ & $2(3)$ & $0_{a, b}$ & $6(10)_{a}$ & $\mathrm{O}_{\mathrm{b}}$ & $2(2)_{a, b}$ \\
\hline LCSD & $1(0.2)$ & $1(0.4)$ & 0 & $1(1)$ & 0 & 0 & 0 & $1(2)$ & 0 & 0 \\
\hline$C E$ & $69(11)_{1}$ & $43(18)_{2}$ & $8(40)_{a}$ & $10(14)_{a, b}$ & $44(10)_{b}$ & $7(10)_{b}$ & $1(4)_{a}$ & $25(41)_{b}$ & $9(12)_{a}$ & $7(8)_{a}$ \\
\hline Syncope $^{d}$ & $62(10)_{1}$ & $39(16)_{2}$ & $7(35)_{a}$ & $8(11)_{a, b}$ & $40(9)_{b}$ & $7(10)_{b}$ & $1(4)_{a}$ & $22(36)_{b}$ & $9(12)_{a}$ & $7(8)_{a}$ \\
\hline$A C A^{e}$ & $4(1)$ & $4(2)$ & 0 & $1(1)$ & $3(1)$ & 0 & 0 & $3(5)$ & $1(1)$ & 0 \\
\hline $\operatorname{SCD}^{f}$ & $4(1)$ & $3(1)$ & 0 & $1(1)$ & $3(1)$ & 0 & 0 & $3(5)$ & 0 & 0 \\
\hline CE without BB & $55(10)_{a}$ & $36(16)_{b}$ & $5(25)_{a}$ & $7(10)_{a, b}$ & $36(8)_{b}$ & $7(10)_{a, b}$ & $1(4)_{a}$ & $20(33)_{b}$ & $8(11)_{a}$ & $7(8)_{a}$ \\
\hline CE with BB & $16(9)$ & $9(14)$ & $3(27)$ & $3(10)$ & $10(8)$ & 0 & 0 & $7(22)$ & $2(14)$ & 0 \\
\hline CE age, $y^{g}$ & $26.1 \pm 5.9_{1}$ & $24.0 \pm 5.72$ & $29.5 \pm 5.7$ & $25.6 \pm 7.0$ & $25.6 \pm 5.7$ & $25.5 \pm 5.5$ & $18.2 \pm 0$ & $23.8 \pm 5.5$ & $25.6 \pm 7.0$ & $23.6 \pm 4.9$ \\
\hline CE before age 18 & $74(12)$ & $30(12)$ & $6(30)_{a}$ & $17(24)_{a}$ & $46(10)_{b}$ & $5(7)_{\mathrm{b}}$ & $1(4)$ & $15(25)$ & $6(8)$ & $8(9)$ \\
\hline
\end{tabular}

Parameters shown as $\mathrm{n}(\%)$, or mean $\pm \mathrm{SD}$

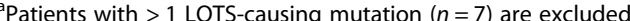

${ }^{b}$ Subscript numbers (1 or 2$)$ indicate that the LQT1 and LQT2 patients have statistically significant difference $(p<0.05)$

'Subscript letters ( $a, b, c$ or d) indicate that at least one group differs from the other three groups as tested separately within LQT1 and LQT2 patient groups.

Groups with different subscript letters ( $a, b, c$ or $d)$ have statistically significant difference after Bonferroni correction $(p<0.05)$

${ }^{\mathrm{d}}$ Triggered by swimming, sport, loud noise or startle

${ }^{\mathrm{e}} \mathrm{A}$ resuscitation that required external defibrillation

f Not explained by any other cause and abrupt in onset if witnessed

${ }^{9}$ The first cardiac event at the age of $18-40$ years

$A C A$ : aborted cardiac arrest, $B B$ : $\beta$-blocker, CE: cardiac event, FF: Finnish founder, ICD: implantable cardioverter-defibrillator, $L C S D$ : left cardiac sympathetic denervation, SCD: sudden cardiac death, SD: standard deviation 
events were fatal. Female mutation carriers were more often probands ( $26 \%$ vs $15 \%, p=0.001)$, and had a longer QTc (473 vs $454 \mathrm{~ms}, p<0.001)$ as compared with males. Altogether, QTc duration $\geq 500 \mathrm{~ms}$ was measured in 132 (15\%) mutation carriers. One family had 7, three families had 3, and the remaining families had $0-2$ cardiac events.

Risk factors for cardiac events before $\beta$-blocker treatment LQT2 genotype was associated with a higher risk of cardiac events in comparison to LQT1 (cumulative probability $18 \%$ vs $11 \%, p=0.01$; HR $=2.1, p=0.002$, Table 2 ). Both LQT1 and LQT2 females were more often symptomatic than males (cumulative rate 16\% vs 3\%, $p$ $<0.001$, for LQT1; and $23 \%$ vs $8 \%, p=0.01$, for LQT2, Figure 1), with a hazard ratio of 3.2 for the female versus male comparison $(\mathrm{p}<0.001)$. The risk was distinctly higher in patients who were symptomatic before the age of 18 years (cumulative rate $52 \%$ vs $9 \%, p<0.001$; HR = 5.93, $\mathrm{p}<0.001)$. QTc duration $\geq 500 \mathrm{~ms}$ increased the risk 2.7-fold compared to QTc $<470 \mathrm{~ms}(p=0.001)$. We repeated the analyses after excluding FF mutation carriers, and the results regarding genotype, gender, symptoms before age 18, and QTc duration were similar.

The risk of cardiac events was higher in mutation carriers than in non-carrier relatives even after adjustment for QTc, gender, and cardiac events before age 18 (Table 3). In comparison with non-carrier relatives with QTc < $440 \mathrm{~ms}$, mutation carriers with QTc $<440$ and $\geq 440 \mathrm{~ms}$ had a 4.2 -fold $(p=0.01)$ and an 11.1-fold $(p<0.001)$ risk, respectively, of suffering a cardiac event.

Upon pairwise comparison, KCNQ1 D317N mutation carriers showed a higher risk of cardiac events than G589D, c.1129-2G > A or other KCNQ1 mutation carriers (cumulative probability $40 \%, 10 \%, 11 \%$ and $14 \%$, respectively, $p=0.002-0.047$, Figure 2 ; $\mathrm{HR}=3.0-3.9, p<$ $0.001-0.03)$. Risk or rate of events did not differ between

Table 2 Cox regression model: Adjusted risk of cardiac events at the age of 18-40 years in LQT1 and LQT2 patients before initiation of $\beta$-blocker medication ${ }^{a}$

\begin{tabular}{llll}
\hline & Hazard ratio & $\begin{array}{l}\text { 95\% confidence } \\
\text { interval }\end{array}$ & $P$-value \\
\hline LQT2 vs LQT1 & 2.11 & $1.33-3.34$ & 0.002 \\
Female vs male & 3.18 & $1.71-5.91$ & $<0.001$ \\
CE vs no CE before age 18 & 5.93 & $3.72-9.44$ & $<0.001$ \\
QTC $\geq 500$ vs $<470 \mathrm{~ms}$ & 2.66 & $1.53-4.64$ & 0.001 \\
QTc $\geq 500$ vs 470-499 ms & 2.22 & $1.23-4.00$ & 0.01 \\
QTc 470-499 vs $<470 \mathrm{~ms}$ & 1.20 & $0.71-2.04$ & 0.50 \\
\hline
\end{tabular}

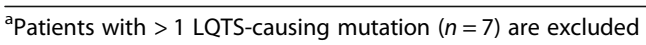

The model was adjusted for family membership using robust

sandwich estimators

A separate QTc missing covariate was used for patients whose QTc data were unavailable $(n=35)$

CE: cardiac event
G589D, c.1129-2G > A and other KCNQ1 mutation carriers. Among LQT2 patients the carriers of the c. 453delC, L552S or R176W mutation had fewer cardiac events than carriers of other $\mathrm{KCNH} 2$ mutations (cumulative rate $5 \%, 13 \%, 9 \%$ and $43 \%$, respectively, $p<0.001-$ 0.013 , Figure 3; $\mathrm{HR}=0.11-0.23, \mathrm{p}<0.001$ ).

An analysis of the effect of the mutation site (Table $1 S$ ) indicated that the cumulative rate of cardiac events was higher in patients carrying a missense KCNQ1 nonFF mutation in pore-loop than in non-pore-loop region $(37 \%$ vs $7 \%, p=0.01)$. In a pairwise comparison, poreloop mutation carriers also had a higher rate of events than carriers of a KCNQ1 G589D or c.1129-2G > A mutation $(p=0.02-0.001)$, but there was no difference between non-pore-loop and FF mutation carriers $(p=0.48-0.53)$. A similar analysis of the LQT2 patients showed that among missense $\mathrm{KCNH} 2$ non-FF mutation carriers there was a tendency of higher event rate in patients with a non-pore-loop than a pore-loop mutation ( $58 \%$ vs $28 \%, p=0.14$ ). Non-pore-loop mutation carriers had a higher event rate than $K C N H 2$ L552S or R176W mutation carriers $(p<0.001)$, and the event rate was similar between carriers of a pore-loop and a FF mutation $(p=0.054-0.15)$.

The rate of cardiac events was the same regardless of whether the mutation had been inherited from mother or father as tested separately among all KCNQ1, KCNQ1 G589D, and all $K C N H 2$ mutation carriers (data were available for $459 \mathrm{KCNQ} 1$ and $150 \mathrm{KCNH} 2$ mutation carriers).

\section{$\beta$-blocker treatment}

$\beta$-blocker medication was initiated to 249 mutation carriers at a mean age of 22.3 and 22.7 years in LQT1 and LQT2 patients, respectively. Use of medication was more common in non-FF than FF patients ( $45 \%$ vs $25 \%, p<0$. 001 ), and in females than males ( $34 \%$ vs $20 \%, p<0.001$ ). Altogether 27 patients suffered a cardiac event during the medication, including SCD in four patients. Breakthrough events were more common in non-FF than FF mutation carriers $(16 \%$ vs $7 \%, p=0.04)$. In patients to whom $\beta$ blocker was prescribed, the medication was associated with $60-81 \%$ reduction in the risk of first cardiac event at the age of $18-40$ years $(\mathrm{p}<0.001$, Table 4$)$.

Non-compliance to $\beta$-blocker medication associated with a 1.9-fold $(p<0.001)$ increase in the risk of cardiac events. However, the incidence rates of cardiac events in noncompliant patients were 52.0 and 34.0 per 1000 person-years before and after initiation of the medication, respectively, indicating a protective impact also in these patients.

\section{Concomitant medications}

Treatment with psychotropic agents was equally common in mutation carriers and non-carrier relatives 


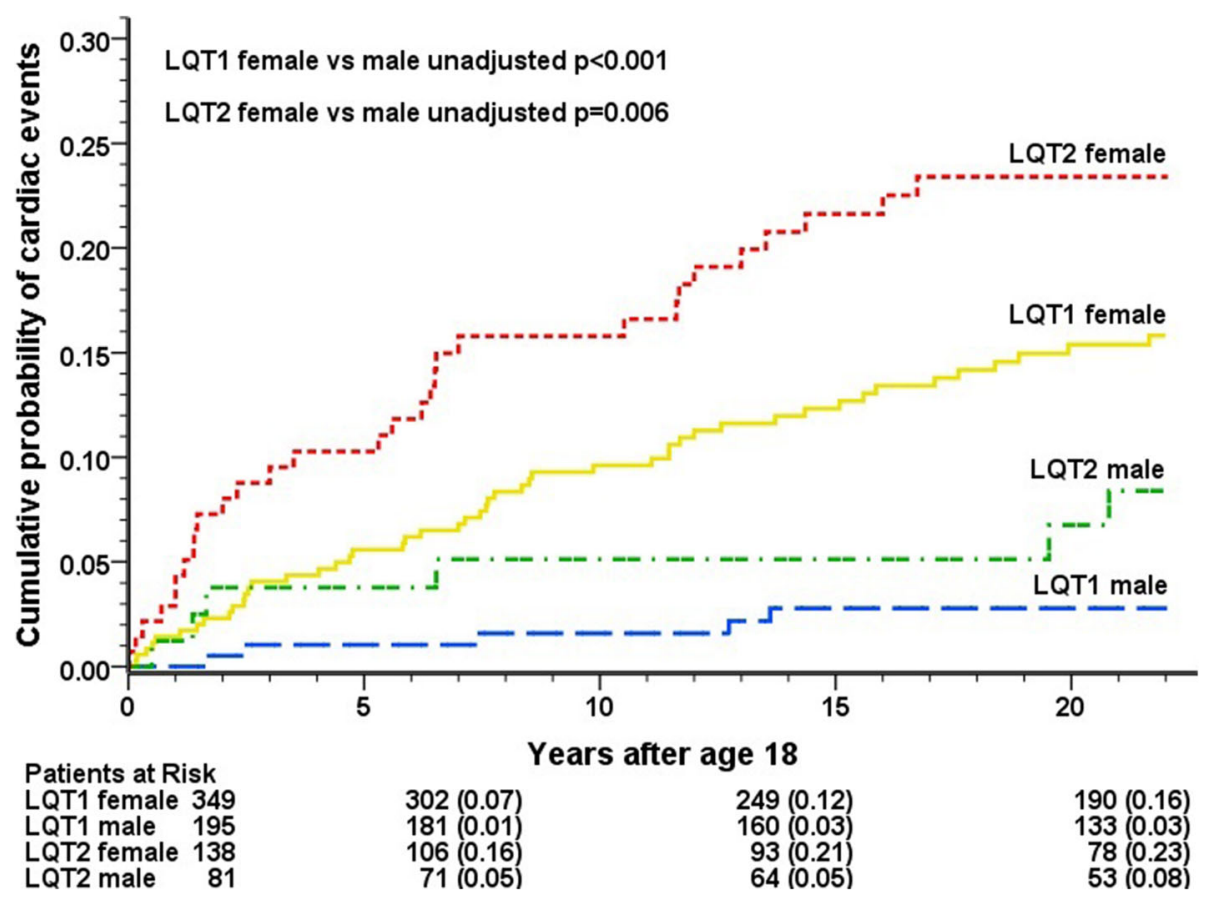

Fig. 1 Cumulative incidence of cardiac events in LQT1 and LQT2 patients by gender before initiation of $\beta$-blocker treatment at the age of 18-40 years

(antidepressants: $5 \%$ vs $5 \%, p=1.00$; antipsychotics: $2 \%$ vs $1 \%, p=1.00$; anxiolytics: $0.4 \%$ vs $0.6 \%, p=1.00$, respectively). However, mutation carriers who had suffered a cardiac event reported more often taking daily antidepressant drug at the end of the follow-up (12\% vs $3 \%$, $p=0.02)$.

Table 3 Cox regression model: Adjusted risk of cardiac events at the age of 18-40 years in mutation carriers and non-carrier relatives before initiation of $\beta$-blocker medication ${ }^{a}$

\begin{tabular}{|c|c|c|c|}
\hline & Hazard ratio & $\begin{array}{l}95 \% \text { confidence } \\
\text { interval }\end{array}$ & $P$-value \\
\hline Non-carrier ${ }^{\mathrm{b}}$ & 1 (reference) & - & - \\
\hline KCNQ1 G589D & 6.06 & $3.49-10.5$ & $<0.001$ \\
\hline KCNQ1 C.1129-2A > G & 6.83 & $3.19-14.6$ & $<0.001$ \\
\hline KCNQ1, other mutations ${ }^{c}$ & 8.02 & $3.43-18.8$ & $<0.001$ \\
\hline KCNQ1 D317N & 23.7 & $11.0-51.1$ & $<0.001$ \\
\hline KCNH2 c.453delC & 3.86 & $1.91-7.79$ & $<0.00$ \\
\hline KCNH2 R176W & 5.87 & $2.89-11.9$ & $<0.001$ \\
\hline KCNH2 L552S & 7.80 & $3.86-15.8$ & $<0.0$ \\
\hline$K C N H 2$, other mutations ${ }^{c}$ & 33.3 & $18.4-60.3$ & $<0.0$ \\
\hline
\end{tabular}

${ }^{\mathrm{a}}$ Patients with $>1$ LQTS-causing mutation $(n=7)$ are excluded

${ }^{b}$ Mean QTc was significantly longer in all eight mutation carrier groups mentioned in the Table 3 than in non-carrier relatives (QTC $424 \pm 26$ and 410

$\pm 22 \mathrm{~ms}$ in female and male non-carriers, respectively). The cumulative probability of cardiac events was $2 \%$ in non-carrier relatives

'The KCNQ1 and KCNH2 mutations in the study are listed in Additional file 2: Table $\mathrm{S} 1$

The model was adjusted for gender, QTc duration, cardiac events before age 18 , and family membership

\section{Device therapy and LCSD}

Characteristics of the 39 patients with ICD, pacemaker or LCSD are detailed in Additional file 2: Table S4. The incidence rate of cardiac events showed reduction after ICD implantation: 152.4 and 56.8 per 1000 person-years before and after implantation, respectively. Similarly, cardiac events decreased after pacemaker implantation: 82.3 and 0 per 1000 person-years. ICD was implanted more frequently to non-FF than FF mutation carriers (7\% vs $1 \%, p<0.001)$. Common ICD implantation indications were ACA, or LQTS-related syncope during $\beta$-blocker medication. An appropriate ICD shock therapy occurred in seven (32\%) and an inappropriate shock in three (14\%) patients. Six patients suffered a complication in the ICD or pacemaker system (incidence rate 26. 8 per 1000 person-years).

\section{Triggers and predisposing factors for SCD and ACA}

The patients with SCD, ACA, or ICD shock therapy are presented in Table 5. Common factors predisposing to SCD were QT-prolonging medication ( $n=4$; citalopram, thioridazine or tizanidine), and absence of $\beta$-blocker therapy $(n=3)$. Only one $\beta$-blocker medication -compliant patient without predisposing factors suffered a SCD. Of the eight patients who suffered ACA, two were using a QT-prolonging drug (terfenadine or amiodarone), and none were on $\beta$-blocker medication at the time of the event. 


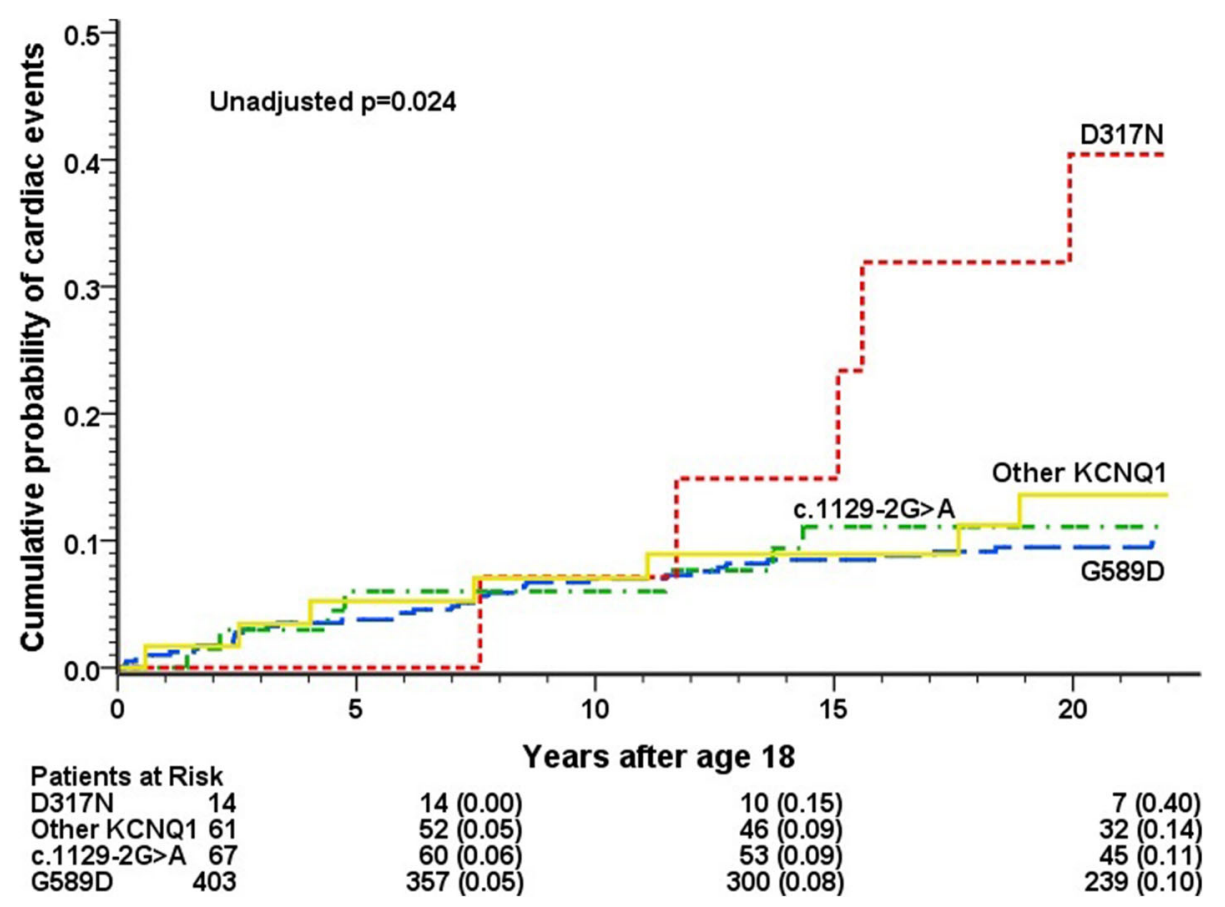

Fig. 2 Cumulative incidence of cardiac events in LQT1 patients by mutation before initiation of $\beta$-blocker treatment at the age of 18-40 years

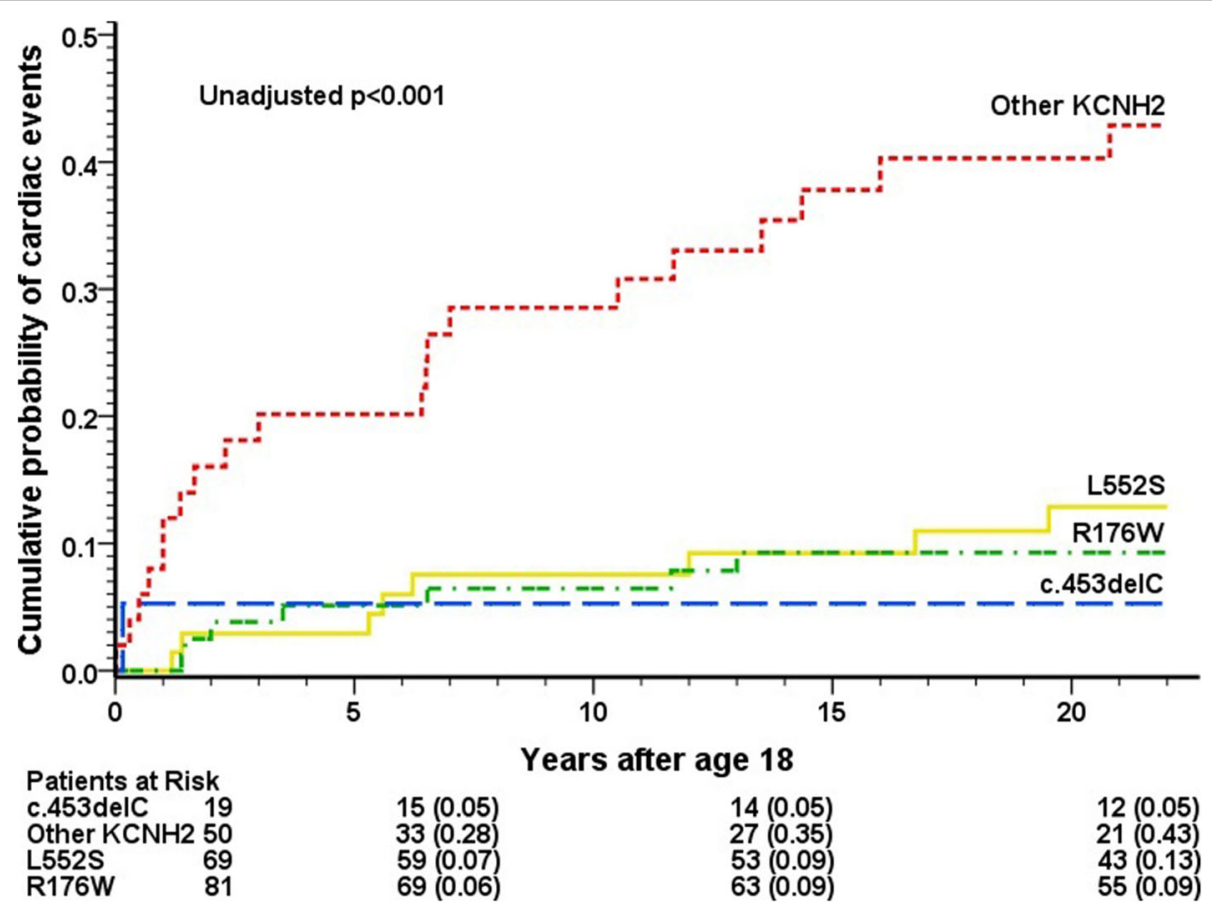

Fig. 3 Cumulative incidence of cardiac events in LQT2 patients by mutation before initiation of $\beta$-blocker treatment at the age of 18-40 years 
Table 4 Time-dependent Cox regression model: Adjusted risk factors for cardiac events at the age of 18-40 years in the 249 LQT1 and LQT2 patients who were treated with $\beta$-blocker medication $^{a}$

\begin{tabular}{llll}
\hline & Hazard ratio & $\begin{array}{l}\text { 95\% confidence } \\
\text { interval }\end{array}$ & P-value \\
\hline BB vs no BB in non-FF & 0.40 & $0.29-0.57$ & $<0.001$ \\
BB vs no BB in KCNQ1 G589D & 0.19 & $0.09-0.41$ & $<0.001$ \\
BB vs no BB in other FF & 0.30 & $0.18-0.51$ & $<0.001$ \\
Non-compliance vs compliance & 1.87 & $1.35-2.59$ & $<0.001$ \\
Side effects vs no side effects & 1.08 & $0.80-1.47$ & 0.61 \\
\hline
\end{tabular}

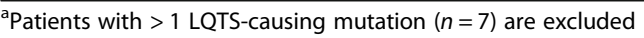
$\beta$-blocker treatment was considered in a time-dependent manner The effect of $\beta$-blocker treatment is shown separately for carriers of non-FF, KCNQ1 G589D, or other FF mutation

The $\beta$-blockers used were bisoprolol (43\%), propranolol (33\%), atenolol (12\%), metoprolol (10\%), acebutolol ( $2 \%)$, and betaxolol (1\%)

The model was adjusted for gender, QTc duration, cardiac events before age 18 , and family membership

A separate QTc missing covariate was used for patients whose QTc data were unavailable

$\mathrm{BB}=\beta$-blocker

\section{Discussion}

The present study explored the clinical course of LQTS in 867 adult KCNQ1 and KCNH2 mutation carriers and evaluated the risk in six specific mutations. To our knowledge, this is the largest LQTS study of genotyped subjects examining the clinical course in the absence of $\beta$-blocker medication, and the first to investigate the clinical course without $\beta$-blocker therapy in adult LQTS population.

\section{Risk factors for cardiac events}

Similar to previous studies, female gender, LQT2 genotype, cardiac events before the age of 18 , and prolonged QTc duration were found to increase the risk of cardiac events. [7, 8, 10, 11, 17-19, 22] In the present study, mutation carriers with a normal QTc duration (< $440 \mathrm{~ms}$ ) had a 4.2-fold risk compared to non-carrier relatives, whereas in an earlier study the corresponding risk was 10-fold. [4] A potential reason for the difference is the end point of ACA or SCD in the previous study, as opposed to LQTS-related syncope, ACA, ICD shock, or SCD in our study. An earlier study demonstrated that cardiac events among genotype-negative family members are mostly attributed to nonfatal syncopal episodes. [23] Although mutation carriers with a normal QTc had a higher risk than non-carrier relatives, normal QTc associated with a good prognosis even in patients left untreated with $\beta$-blockers: none of the previously asymptomatic non-proband mutation carriers suffered a cardiac event during the prospective follow-up. This is of note as cascade screening of family members reveals a growing number of asymptomatic mutation carriers with a normal or only slightly prolonged QTc.

\section{Association of the mutation type with clinical events} In the current study, missense KCNQ1 mutations located in the pore-loop region associated with a higher rate of cardiac events than non-pore-loop mutations, which was not seen in a previous study. [24] However, in the present study most of the pore-loop mutation carriers had the highly malign $K C N Q 1$ D317N mutation. Previously, KCNQ1 cytoplasmic loop (c-loop) mutations have been associated with a higher risk. [11] In our study, only six patients were carriers of a c-loop mutation precluding exact comparison of c-loop and non-cloop mutations. In the present study, $\mathrm{KCNH} 2$ mutations situated in the pore-loop region were not associated with an increased risk as seen in previous studies. [10, 22] On the other hand, only 15 subjects had a pore-loop mutation in our analysis.

All four founder mutations had a significant QTprolonging effect and associated with increased risk of cardiac events. Similarly to our recent study of pediatric LQT1 and LQT2 population, the $K C N H 2$ FF mutations led to a milder phenotype than non-FF $K C N H 2$ mutations. [14] This might be related to the fact that both $\mathrm{KCNH} 2 \mathrm{FF}$ mutations lead to a functional channel with increased deactivation rate, $[21,25]$ whereas many nonFF $\mathrm{KCNH} 2$ mutations have more dramatic effects on channel function. However, the risk between KCNQ1 FF and non-FF mutations did not differ from each other after excluding the KCNQ1 D317N mutation.

The KCNQ1 D317N mutation appeared exceptionally malign, and previously it has been shown to associate with diminished chronotropic response and exaggerated QTc prolongation after exercise. [26] This mutation is located in the pore-loop region and leads to complete loss of channel function with a dominant negative effect on the wild type channel protein. [27] On the other hand, the phenotype associated with the $K C N H 2$ c. 453delC mutation turned out to be reasonably mild, in harmony with an earlier study. [28] This N-terminal mutation leads to a premature termination codon, which likely targets the mutated mRNA to non-sense mediated mRNA decay without any dominant negative effect.

\section{Medical treatment}

Only $29 \%$ of the patients in the present study used $\beta$-blocker medication compared to $45-62 \%$ in earlier studies. [10, 11, 19, 22] However, the previous studies included adolescents, who are more frequently treated with $\beta$-blockers. [29] In accordance with previous studies, non-compliance to $\beta$-blocker therapy increased the risk of cardiac events. [14, 30] Nevertheless, also noncompliant patients demonstrated a decrease in the incidence rate of cardiac events after initiating medication, which suggests a protective role for $\beta$-blockers, even when present in suboptimal therapeutic concentrations. 
Table 5 SCD, ACA and ICD shock cases

\begin{tabular}{|c|c|c|c|c|c|c|c|}
\hline Case & Mutation & $\begin{array}{l}\text { Age at } \\
\text { event }\end{array}$ & $\beta$-blocker & Trigger or predisposing factor & $\begin{array}{l}\text { LQTS dg } \\
\text { before event }\end{array}$ & $\begin{array}{l}\text { Device } \\
\text { implantation }^{\mathrm{a}} \\
\end{array}$ & $\begin{array}{l}\text { CE before SCD, } \\
\text { ACA or ICD shock }\end{array}$ \\
\hline \multicolumn{8}{|l|}{ SCD } \\
\hline 1 & KCNQ1 G589D & 35.8 & $\begin{array}{l}\text { No (non- } \\
\text { compliance) }\end{array}$ & Unwitnessed & Yes & No & No \\
\hline 2 & KCNQ1 G589D & 35.0 & Yes & Unwitnessed, citalopram, ethanol & Yes & No & Syncope (no BB) \\
\hline 3 & KCNQ1 C.1032G > A & 32.0 & Yes & Physical exertion, citalopram, ethanol & Yes & No & Syncope (no BB) \\
\hline 4 & KCNQ1 G589D & 31.3 & Yes & Awakening, thioridazine & Yes & No & Syncope (no BB) \\
\hline 5 & KCNH2 Y569H & 25.5 & $\begin{array}{l}\text { No (non- } \\
\text { compliance) }\end{array}$ & Awakening, tizanidine, amphetamine & Yes & No & No \\
\hline 6 & KCNH2 c.842dupG & 24.4 & Yes & Alarm clock & Yes & No & Syncope (no BB) \\
\hline 7 & KCNH2 A558E & 21.8 & No & Awakening & No & No & Syncope (no BB) \\
\hline \multicolumn{8}{|l|}{$\mathrm{ACA}^{\mathrm{c}}$} \\
\hline 8 & KCNH2 c.643delG & 38.8 & No & No trigger & No & ICD (39.0) & No \\
\hline 9 & KCNQ1 G589D & 34.5 & No & Terfenadine, ketoconazole & No & No & Syncope (no BB) \\
\hline 10 & KCNH2 P451L & 31.5 & No & Excitement & No & PM (31.6) & No \\
\hline 11 & KCNQ1 R518Ter & 25.5 & No & Rest & No & No & No \\
\hline $12^{d}$ & KCNH2 L552S & 23.6 & No & Awakening, amiodarone & No & ICD (23.6) & Syncope (no BB) \\
\hline 13 & KCNH2 c.1558-1G > C & 23.5 & No & Rest & No & ICD (23.5) & Syncope (no BB) \\
\hline 14 & KCNQ1 G589D & 21.0 & No & Sport & No & ICD (21.0) & No \\
\hline 15 & KCNQ1 G589D & 20.5 & No & Post partum period, hypokalemia & No & ICD (20.5) & No \\
\hline \multicolumn{8}{|c|}{ ICD shock } \\
\hline 16 & KCNQ1 D317N & 31.1 & Yes & Excitement & Yes & $\mathrm{ICD}(17.2)$ & Syncope (BB) \\
\hline 17 & KCNQ1 G589D & 28.3 & Yes & Excitement & Yes & ICD (26.9) & Syncope (no BB) \\
\hline $12^{\mathrm{d}}$ & KCNH2 L552S & 25.6 & Yes & NA & Yes & ICD (23.6) & $\mathrm{ACA}$ (no BB) \\
\hline 18 & KCNH2 W497Ter & 24.0 & $\begin{array}{l}\text { No (non- } \\
\text { compliance) }\end{array}$ & Mirtazapine & Yes & $\mathrm{ICD}(16.0)$ & Syncope (BB) \\
\hline 19 & KCNH2 A561V & 21.4 & Yes & Rest & Yes & ICD (13.1) & $\begin{array}{l}A C A(B B),{ }^{e} I C D \text { shock } \\
(B B)^{e}\end{array}$ \\
\hline $20^{f}$ & $\begin{array}{l}\text { KCNH2 L552S KCNH2 } \\
\text { L552S }\end{array}$ & 20.2 & $\begin{array}{l}\text { No (non- } \\
\text { compliance) }\end{array}$ & $\begin{array}{l}\text { Pneumonia, disturbance of diabetes } \\
\text { treatment }\end{array}$ & Yes & ICD (15.6) & $\begin{array}{l}\text { Syncope (BB), ICD } \\
\text { shock (BB) }^{\mathrm{e}}\end{array}$ \\
\hline 21 & KCNH2 L552S & 19.9 & Yes & Rest & Yes & ICD (18.9) & Syncope (BB) \\
\hline
\end{tabular}

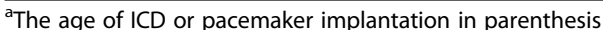

byncope was triggered by swimming, sport, loud noise or startle. "BB" and "no BB" denote patient was and was not, respectively, using $\beta$-blocker at the time of the cardiac event

${ }^{\mathrm{A}} \mathrm{A}$ resuscitation that required external defibrillation

${ }^{\mathrm{d}}$ Case 12 suffered both ACA and ICD shock

${ }^{\mathrm{e}} \mathrm{ACA}$ or ICD shock before the age of 18 years

Homozygous mutation carrier

CE: cardiac event, $d g$ : diagnosis, NA: not available, PM: pacemaker, other abbreviations as in Table 1

However, considerable proportion of the SCD and ACA cases associated with insufficient $\beta$-blocker medication underlining the importance of uninterrupted use of prescribed $\beta$-blockers.

According to recent ESC guidelines, $\beta$-blocker treatment should be initiated if QTc is prolonged, and it may be useful even with normal QTc duration. [3, 4] The results of the current study indicate that asymptomatic adult LQT1 and LQT2 males with FF mutation and QTc duration $<500 \mathrm{~ms}$ have a very low risk, and suggest that the avoidance of risk factors may be a sufficient measure.
Also, $\beta$-blocker medication might not be mandatory for primary prevention in adult $\mathrm{KCNH} 2 \mathrm{c} .453 \mathrm{delC}$ mutation carriers and female FF mutation carriers with QTc < $500 \mathrm{~ms}$. For the remaining patients $\beta$-blocker treatment is recommended.

In the current study, the use of antidepressant drugs at the end of the follow-up was more common in symptomatic patients. Analysis of a possible causal connection was not feasible due to limited data on the length of the antidepressant medication. However, it is possible that these patients were treated with antidepressants 
already at the time of the cardiac event. Furthermore, four of the seven SCD cases and two of the eight ACA cases involved treatment with a potentially QT-prolonging drug. Therefore, the present and a previous study [30] emphasize avoidance of QT-prolonging drugs in prevention of potentially life-threatening cardiac events.

\section{Device therapy}

The incidence rate of cardiac events showed reduction after ICD or pacemaker implantation. A potential explanation arises from bradycardia pacing which has been previously demonstrated to reduce the risk of ICD shocks in high-risk patients. [5] However, in the current study, the initiation of $\beta$-blocker therapy was coincidental with device implantation in 18 of the 37 cases. Therefore, the reduction in cardiac events might be attributable to $\beta$-blocker treatment.

\section{Study limitations}

There are a number of noteworthy limitations in our study. First, only $55 \%$ of the patients initially surveyed responded to our inquiry leading to a possibility of selection bias. Second, comparison of patients with different mutation categories occasionally resulted in relatively small patient subgroups. Third, initiation of $\beta$-blocker medication was not standardized across participants leading to a concern about confounding by indication. Fourth, we have performed rather many statistical comparisons which may increase the number of false positive findings.

\section{Conclusions}

Molecularly defined LQT1 and LQT2 patients who survive till adulthood continue to be at risk of cardiac events. The clinical risk factors for cardiac events in patients without $\beta$-blocker medication were mostly found to be similar to those reported in previous studies that included patients treated with $\beta$-blockers. Specific KCNQ1 and KCNH2 mutations were associated with varied risk of cardiac events, independently of gender, QTc duration, and cardiac events before the age of 18 . The identification of high-risk and low-risk mutations may enhance risk stratification, and may help to reveal patient groups in which lifestyle modifications are a sufficient measure.

\section{Additional files}

Additional file 1: Questionnaire. (DOCX $20 \mathrm{~kb}$ )

Additional file 2: Table S1. Mutations in the study. Table S2.

Characteristics of the patients with $>1$ mutation. Table S3. Characteristics of

the non-carrier relatives. Table S4. ICDs, pacemakers and LCSDs. (DOC 233 kb)

\section{Abbreviations}

ACA: Aborted cardiac arrest; BB: B-blocker; FF: Finnish founder; HR: Hazard ratio; ICD: Implantable cardioverter-defibrillator; LCSD: Left cardiac sympathetic denervation; LQT1: Long QT syndrome type 1; LQT2: Long QT syndrome type 2; LQTS: Long QT syndrome; SCD: Sudden cardiac death

\section{Acknowledgements}

We thank Minna Härkönen, Susanna Saarinen, and IImari Määttänen for their excellent technical assistance.

\section{Funding}

This work was supported by The Sigrid Juselius Foundation; and The Finnish Foundation for Cardiovascular Research.

\section{Availability of data and materials}

The datasets used and/or analysed during the current study are available from the corresponding author on reasonable request.

\section{Authors' contributions}

MK, AM and HS made substantial contribution to the design of the study. MK collected, analysed and interpreted the data, and drafted the manuscript ASH provided senior supervision for statistical analyses. AMT contributed to the classification of mutations and polymorphisms. All authors (MK, AM, HS, ASH, AMT, VS, PJLF, KP, LT, KK, and MV) made substantial contribution to interpretation of data, critically reviewed the manuscript for important intellectual content, gave approval for its final version to be published, and agreed to be accountable for all aspects of the work. All authors have read and approved the manuscript.

Ethical approval and consent to participate

Participants signed an informed consent. The Ministry of Social Affairs and Health consented for the participation of the deceased subjects. The study was approved by the Ethical Review Committee of Helsinki University Central Hospital.

\section{Consent for publication}

Not applicable.

\section{Competing interests}

The authors declare that they have no competing interests.

\section{Publisher's Note}

Springer Nature remains neutral with regard to jurisdictional claims in published maps and institutional affiliations.

\section{Author details}

${ }^{1}$ Heart and Lung Center, Helsinki University Central Hospital, Helsinki, Finland. ${ }^{2}$ Department of Health, National Institute for Health and Welfare, Helsinki, Finland. ${ }^{3}$ Department of Medicine, Helsinki University Central Hospital and University of Helsinki, Helsinki, Finland. ${ }^{4}$ synlab Humane Genetik Munich, Munich, Germany. ${ }^{5}$ Department of Genetics, United Medix Laboratories Ltd, Helsinki, Finland.

Received: 23 July 2017 Accepted: 23 March 2018

Published online: 05 April 2018

References

1. Schwartz PJ, Periti M, Malliani A. The long Q-T syndrome. Am Heart J. 1975; 89:378-90.

2. Schwartz PJ, Ackerman MJ, George AL Jr, et al. Impact of genetics on the clinical management of channelopathies. J Am Coll Cardiol. 2013;62:169-80.

3. Priori SG, Blomstrom-Lundqvist C, Mazzanti A, et al. 2015 ESC Guidelines for the management of patients with ventricular arrhythmias and the prevention of sudden cardiac death: The Task Force for the Management of Patients with Ventricular Arrhythmias and the Prevention of Sudden Cardiac Death of the European Society of Cardiology (ESC) Endorsed by: Association for European Paediatric and Congenital Cardiology (AEPC). Europace. 2015;17:1601-87.

4. Goldenberg I, Horr S, Moss AJ, et al. Risk for life-threatening cardiac events in patients with genotype-confirmed long-QT syndrome and normal-range corrected QT intervals. J Am Coll Cardiol. 2011;57:51-9.

5. Schwartz PJ, Spazzolini C, Priori SG, et al. Who are the long-QT syndrome patients who receive an implantable cardioverter-defibrillator and what happens to them?: data from the European Long-QT Syndrome Implantable Cardioverter-Defibrillator (LQTS ICD) Registry. Circulation. 2010;122:1272-82. 
6. Zareba W, Moss AJ, Daubert JP, et al. Implantable cardioverter defibrillator in high-risk long QT syndrome patients. J Cardiovasc Electrophysiol. 2003;14:337-41.

7. Priori SG, Schwartz PJ, Napolitano C, et al. Risk stratification in the long-QT syndrome. N Engl J Med. 2003;348:1866-74.

8. Zareba W, Moss AJ, Schwartz PJ, et al. Influence of genotype on the clinical course of the long-QT syndrome. International Long-QT Syndrome Registry Research Group. N Engl J Med. 1998;339:960-5.

9. Schwartz PJ, Priori SG, Spazzolini C, et al. Genotype-phenotype correlation in the long-QT syndrome: gene-specific triggers for life-threatening arrhythmias. Circulation. 2001;103:89-95.

10. Migdalovich D, Moss AJ, Lopes CM, et al. Mutation and gender-specific risk in type 2 long QT syndrome: implications for risk stratification for lifethreatening cardiac events in patients with long QT syndrome. Heart Rhythm. 2011:8:1537-43.

11. Costa J, Lopes CM, Barsheshet A, et al. Combined assessment of sex- and mutation-specific information for risk stratification in type 1 long QT syndrome. Heart Rhythm. 2012;9:892-8.

12. Liu JF, Moss AJ, Jons $C$, et al. Mutation-specific risk in two genetic forms of type 3 long QT syndrome. Am J Cardiol. 2010;105:210-3.

13. Crotti L, Spazzolini C, Schwartz PJ, et al. The common long-QT syndrome mutation KCNQ1/A341V causes unusually severe clinical manifestations in patients with different ethnic backgrounds: toward a mutation-specific risk stratification. Circulation. 2007;116:2366-75.

14. Koponen M, Marjamaa A, Hiippala A, et al. Follow-Up of 316 Molecularly Defined Pediatric Long-QT Syndrome Patients: Clinical Course, Treatments and Side Effects. Circ Arrhythm Electrophysiol. 2015;8:815-23.

15. Itoh H, Dochi K, Shimizu W, et al. A Common Mutation of Long QT Syndrome Type 1 in Japan. Circ J. 2015;79:2026-30.

16. Stattin EL, Bostrom IM, Winbo A, et al. Founder mutations characterise the mutation panorama in 200 Swedish index cases referred for Long QT syndrome genetic testing. BMC Cardiovasc Disord. 2012;12:95-2261. 12-95

17. Priori SG, Napolitano C, Schwartz PJ, et al. Association of long QT syndrome loci and cardiac events among patients treated with beta-blockers. JAMA. 2004;292:1341-4.

18. Sauer AJ, Moss AJ, McNitt S, et al. Long QT syndrome in adults. J Am Coll Cardiol. 2007:49:329-37.

19. Goldenberg I, Bradley J, Moss A, et al. Beta-blocker efficacy in highrisk patients with the congenital long-QT syndrome types 1 and 2 : implications for patient management. J Cardiovasc Electrophysiol. 2010;21:893-901.

20. Fodstad $H$, Swan $H$, Laitinen $P$, et al. Four potassium channel mutations account for $73 \%$ of the genetic spectrum underlying long-QT syndrome (LQTS) and provide evidence for a strong founder effect in Finland. Ann Med. 2004;36(Suppl 1):53-63.

21. Fodstad H, Bendahhou S, Rougier JS, et al. Molecular characterization of two founder mutations causing long QT syndrome and identification of compound heterozygous patients. Ann Med. 2006;38:294-304.

22. Shimizu W, Moss AJ, Wilde AA, et al. Genotype-phenotype aspects of type 2 long QT syndrome. J Am Coll Cardiol. 2009;54:2052-62.

23. Barsheshet A, Moss AJ, McNitt S, et al. Risk of syncope in family members who are genotype-negative for a family-associated long-QT syndrome mutation. Circ Cardiovasc Genet. 2011:4:491-9.

24. Moss AJ, Shimizu W, Wilde AA, et al. Clinical aspects of type-1 long-QT syndrome by location, coding type, and biophysical function of mutations involving the KCNQ1 gene. Circulation. 2007;115:2481-9.

25. Piippo K, Laitinen $\mathrm{P}$, Swan $\mathrm{H}$, et al. Homozygosity for a HERG potassium channel mutation causes a severe form of long QT syndrome: identification of an apparent founder mutation in the Finns. J Am Coll Cardiol. 2000;35:1919-25.

26. Swan $H$, Viitasalo $M$, Piippo $K$, et al. Sinus node function and ventricular repolarization during exercise stress test in long QT syndrome patients with KVLQT1 and HERG potassium channel defects. J Am Coll Cardiol. 1999;34:823-9.

27. Wollnik B, Schroeder BC, Kubisch C, et al. Pathophysiological mechanisms of dominant and recessive KVLQT1 K+ channel mutations found in inherited cardiac arrhythmias. Hum Mol Genet. 1997;11:1943-9.

28. Laitinen $\mathrm{P}$, Fodstad $\mathrm{H}$, Piippo $\mathrm{K}$, et al. Survey of the coding region of the HERG gene in long QT syndrome reveals six novel mutations and an amino acid polymorphism with possible phenotypic effects. Hum Mutat. 2000;15:580-1.
29. Liu JF, Jons C, Moss AJ, et al. Risk factors for recurrent syncope and subsequent fatal or near-fatal events in children and adolescents with long QT syndrome. J Am Coll Cardiol. 2011;57:941-50.

30. Vincent GM, Schwartz PJ, Denjoy I, et al. High efficacy of beta-blockers in long-QT syndrome type 1: contribution of noncompliance and QTprolonging drugs to the occurrence of beta-blocker treatment "failures". Circulation. 2009;119:215-21.

\section{Submit your next manuscript to BioMed Central and we will help you at every step:}

- We accept pre-submission inquiries

- Our selector tool helps you to find the most relevant journal

- We provide round the clock customer support

- Convenient online submission

- Thorough peer review

- Inclusion in PubMed and all major indexing services

- Maximum visibility for your research

Submit your manuscript at www.biomedcentral.com/submit 